\title{
乌ु
}

\section{Hamiltonian for the Zeros of the Riemann Zeta Function}

\author{
Carl M. Bender, ${ }^{1}$ Dorje C. Brody, ${ }^{2,3}$ and Markus P. Müller ${ }^{4,5}$ \\ ${ }^{1}$ Department of Physics, Washington University, St. Louis, Missouri 63130, USA \\ ${ }^{2}$ Department of Mathematics, Brunel University London, Uxbridge UB8 3PH, United Kingdom \\ ${ }^{3}$ Department of Optical Physics and Modern Natural Science, St. Petersburg National Research University \\ of Information Technologies, Mechanics and Optics, St. Petersburg 197101, Russia \\ ${ }^{4}$ Departments of Applied Mathematics and Philosophy, University of Western Ontario, Middlesex College, \\ London, Ontario N6A 5B7, Canada \\ ${ }^{5}$ The Perimeter Institute for Theoretical Physics, Waterloo, Ontario N2L 2Y5, Canada
}

(Received 23 September 2016; revised manuscript received 17 February 2017; published 30 March 2017)

A Hamiltonian operator $\hat{H}$ is constructed with the property that if the eigenfunctions obey a suitable boundary condition, then the associated eigenvalues correspond to the nontrivial zeros of the Riemann zeta function. The classical limit of $\hat{H}$ is $2 x p$, which is consistent with the Berry-Keating conjecture. While $\hat{H}$ is not Hermitian in the conventional sense, $i \hat{H}$ is $\mathcal{P} \mathcal{T}$ symmetric with a broken $\mathcal{P} \mathcal{T}$ symmetry, thus allowing for the possibility that all eigenvalues of $\hat{H}$ are real. A heuristic analysis is presented for the construction of the metric operator to define an inner-product space, on which the Hamiltonian is Hermitian. If the analysis presented here can be made rigorous to show that $\hat{H}$ is manifestly self-adjoint, then this implies that the Riemann hypothesis holds true.

DOI: 10.1103/PhysRevLett.118.130201

The Riemann zeta function $\zeta(z)$ is conventionally represented as the sum or the integral

$$
\zeta(z)=\sum_{k=1}^{\infty} \frac{1}{k^{z}}=\frac{1}{\Gamma(z)} \int_{0}^{\infty} d t \frac{t^{z-1}}{e^{t}-1} .
$$

(The integral reduces to the sum if the denominator of the integrand is expanded in a geometric series.) Both representations converge and define $\zeta(z)$ as an analytic function when $\operatorname{Re}(z)>1$. These representations diverge when $z=1$ because the zeta function has a simple pole at $z=1$. Substituting $z=-2 n(n=1,2,3, \ldots)$ in the reflection formula

$$
\zeta(z)=2^{z} \pi^{z-1} \sin (\pi z / 2) \Gamma(1-z) \zeta(1-z)
$$

shows that the zeta function vanishes when $z$ is a negativeeven integer. These zeros of $\zeta(z)$ are called the trivial zeros.

The Riemann hypothesis [1] states that the nontrivial zeros of $\zeta(z)$ lie on the line $\operatorname{Re}(z)=\frac{1}{2}$. This hypothesis has attracted much attention for over a century because there is a deep connection with number theory and other branches of mathematics. However, the hypothesis has not been proved or disproved. Any advance in understanding the

Published by the American Physical Society under the terms of the Creative Commons Attribution 4.0 International license. Further distribution of this work must maintain attribution to the author(s) and the published article's title, journal citation, and DOI. zeta function would be of great interest in mathematical science, whether or not one succeeds in finally proving or falsifying the hypothesis.

In this Letter, we examine the Riemann hypothesis by constructing and studying an operator $\hat{H}$ that plays the role of a Hamiltonian. The conjectured property of $\hat{H}$ is that its eigenvalues are exactly the imaginary parts of the nontrivial zeros of the zeta function. The idea that the imaginary parts of the zeros of $\zeta(z)$ might correspond to the eigenvalues of a Hermitian, self-adjoint operator (assuming the validity of the Riemann hypothesis) is known as the Hilbert-Pólya conjecture. Research into this connection has intensified following the observation that the spacings of the zeros of the zeta function on the line $\operatorname{Re}(z)=\frac{1}{2}$ and the spacings of the eigenvalues of a Gaussian unitary ensemble of Hermitian random matrices have the same distribution [2-4]. Berry and Keating conjectured that the classical counterpart of such a Hamiltonian would have the form $H=x p[5,6]$. However, a Hamiltonian possessing this property has hitherto not been found (see [7] for a detailed account of the Berry-Keating program and its extensions).

We propose and consider the Hamiltonian

$$
\hat{H}=\frac{\mathbb{1}}{\mathbb{1}-e^{-i \hat{p}}}(\hat{x} \hat{p}+\hat{p} \hat{x})\left(\mathbb{1}-e^{-i \hat{p}}\right) .
$$

Our main findings are as follows. (i) The non-Hermitian Hamiltonian $\hat{H}$ in (1) formally satisfies the conditions of the Hilbert-Pólya conjecture. That is, if the eigenfunctions of $\hat{H}$ are required to satisfy the boundary condition $\psi_{n}(0)=0$ for all $n$, then the eigenvalues $\left\{E_{n}\right\}$ have the 
property that $\left\{\frac{1}{2}\left(1-i E_{n}\right)\right\}$ are the nontrivial zeros of the Riemann zeta function. (ii) The Hamiltonian $\hat{H}$ reduces to the classical Hamiltonian $H=2 x p$ when $\hat{x}$ and $\hat{p}$ commute, in agreement with the Berry-Keating conjecture. We derive the corresponding boundary condition that leads to the quantization of the Berry-Keating Hamiltonian $\hat{h}^{\mathrm{BK}}=\hat{x} \hat{p}+\hat{p} \hat{x}$. (iii) Although $\hat{H}$ is not Hermitian, $i \hat{H}$ is $\mathcal{P} \mathcal{T}$ symmetric; that is, $i \hat{H}$ is invariant under parity-time reflection (in the sense to be defined), which means that the eigenvalues of $i \hat{H}$ are either real or else occur in complex-conjugate pairs. If $i \hat{H}$ has maximally broken $\mathcal{P} \mathcal{T}$ symmetry - that is, if all of its eigenvalues are pureimaginary complex-conjugate pairs - then the eigenvalues of $\hat{H}$ are real and the Riemann hypothesis follows. (iv) While $\hat{H}$ is not Hermitian (symmetric) with respect to the conventional $\mathcal{L}^{2}$ inner product, we introduce an alternative inner product such that $\langle\hat{H} \varphi, \psi\rangle=\langle\varphi, \hat{H} \psi\rangle$ for all $\varphi(x)$ and $\psi(x)$ belonging to the linear span of the eigenstates of $\hat{H}$. (v) If the Riemann hypothesis is correct, then the eigenvalues of $\hat{H}$ are nondegenerate, and conversely if there are nontrivial roots of $\zeta(z)$ for which $\operatorname{Re}(z) \neq \frac{1}{2}$, then the corresponding eigenvalues and eigenstates are both degenerate.

Preliminaries.-The Hamiltonian $\hat{H}$ in (1) is a similarity transformation of the formally Hermitian local Hamiltonian $\hat{x} \hat{p}+\hat{p} \hat{x}$ via the nonlocal operator $\hat{\Delta}:=\mathbb{1}-e^{-i \hat{p}}$. We must therefore identify properties of the operators $\hat{\Delta}$ and $\hat{\Delta}^{-1}$. We work in units for which $\hbar=1$, so the momentum operator is $\hat{p}=-i \partial_{x}$. Thus, $e^{-i \hat{p}}$ is a shift operator if it acts on functions $f(x)$ that have a Taylor series about $x$ with a radius of convergence greater than one. In this case, $\hat{\Delta}$ is a difference operator:

$$
\hat{\Delta} f(x)=f(x)-f(x-1) .
$$

Because $\hat{\Delta}$ annihilates unit-periodic functions, it does not have an inverse in the space of all smooth functions. However, we shall be interested in functions that vanish as $x \rightarrow \infty$. With this in mind, by taking a series expansion of $\left(\mathbb{1}-e^{-i \hat{p}}\right)^{-1}$ we may define $\hat{\Delta}^{-1}$ as (cf. [8])

$$
\hat{\Delta}^{-1} f(x)=\frac{\mathbb{1}}{i \hat{p}} \sum_{n=0}^{\infty} B_{n} \frac{(-i \hat{p})^{n}}{n !} f(x)
$$

where $\left\{B_{k}\right\}$ are the Bernoulli numbers [9], with the convention that $B_{1}=-\frac{1}{2}$. For some functions $f(x)$ this formal series diverges but it is Borel summable. The operator $(i \hat{p})^{-1}$ is interpreted as an integral operator with a boundary at infinity:

$$
\frac{\mathbb{1}}{i \hat{p}} g(x)=\int_{\infty}^{x} d t g(t)
$$

Then $\hat{\Delta}^{-1}$ defined in (3) has the property that if $f(x)$ vanishes at infinity, then we have $\hat{\Delta}^{-1} \hat{\Delta} f(x)=f(x)$.

Eigenfunctions and eigenvalues. - The solutions to the eigenvalue differential equation $\hat{H} \psi=E \psi$ are given in terms of the Hurwitz zeta function $\psi_{z}(x)=-\zeta(z, x+1)$ on the positive half line $\mathbb{R}^{+}$(the negative sign is our convention), with eigenvalues $i(2 z-1)$. To see this, we multiply the eigenvalue equation $\hat{H} \psi=E \psi$ on the left by $\hat{\Delta}$. This gives a first-order linear differential equation $(\hat{x} \hat{p}+\hat{p} \hat{x}) \hat{\Delta} \psi=E \hat{\Delta} \psi$ for the function $\hat{\Delta} \psi$, whose solution is unique and is given by $\hat{\Delta} \psi=x^{-z}$ for some $z \in \mathbb{C}$, up to a multiplicative constant. To proceed, let us calculate

$$
\begin{aligned}
\hat{\Delta}^{-1} x^{-z} & =\frac{\mathbb{1}}{i \hat{p}} \sum_{n=0}^{\infty} B_{n} \frac{(-i \hat{p})^{n}}{n !}(i \hat{p}) \frac{x^{1-z}}{1-z} \\
& =\frac{1}{1-z} \sum_{n=0}^{\infty} B_{n} \frac{(-i \hat{p})^{n}}{n !} x^{1-z} .
\end{aligned}
$$

Since $i \hat{p}=\partial_{x}$ and $\partial_{x}^{n} x^{\mu}=[\Gamma(\mu+1) / \Gamma(\mu-n+1)] x^{\mu-n}$, we set $\mu=1-z$ to obtain the asymptotic series

$$
\hat{\Delta}^{-1} x^{-z} \sim \frac{\Gamma(2-z)}{1-z} \sum_{n=0}^{\infty} B_{n} \frac{(-1)^{n}}{n !} \frac{x^{1-z-n}}{\Gamma(2-z-n)},
$$

which is valid in the limit as $x \rightarrow \infty$. To obtain the Borel sum [10] of the series, we use the integral representation

$$
\frac{1}{\Gamma(2-z-n)}=\frac{1}{2 \pi i} \int_{C} d u e^{u} u^{n+z-2},
$$

where $C$ denotes a Hankel contour that encircles the negative- $u$ axis in the positive orientation [9]. Hence,

$$
\begin{aligned}
\hat{\Delta}^{-1} x^{-z} & =\frac{\Gamma(1-z)}{2 \pi i} x^{1-z} \int_{C} d u e^{u} u^{z-2} \sum_{n=0}^{\infty} B_{n} \frac{(-u / x)^{n}}{n !} \\
& =\frac{\Gamma(1-z)}{2 \pi i} x^{-z} \int_{C} d u \frac{e^{u} u^{z-1}}{1-e^{-u / x}} .
\end{aligned}
$$

Finally, we let $u / x=t$ and get

$$
\hat{\Delta}^{-1} x^{-z}=\frac{\Gamma(1-z)}{2 \pi i} \int_{C} d t \frac{e^{x t} t^{z-1}}{1-e^{-t}},
$$

which we recognize as the negative of the integral representation for the Hurwitz zeta function [9]. (An analogous result was obtained in a different context in [11].) It follows that $\psi_{z}(x)=-\zeta(z, x+1)$ up to an additive unit-periodic function, but $\hat{H} \psi=E \psi$ implies that the periodic function must be identically zero. We thus deduce that $\psi_{z}(x)=$ $-\zeta(z, x+1)$ is the solution to the eigenvalue differential equation with eigenvalue $i(2 z-1)$ : 


$$
\hat{H} \psi_{z}(x)=\hat{\Delta}^{-1}(\hat{x} \hat{p}+\hat{p} \hat{x}) x^{-z}=i(2 z-1) \psi_{z}(x) .
$$

Next, we impose the boundary condition that $\psi_{z}(0)=0$ on the class of functions $\psi_{z}(x)$ that satisfy the eigenvalue differential equation. This yields a countable set of eigenfunctions of $\hat{H}$. (Since $\hat{H}$ is similar to a first-order differential operator, we impose just one boundary condition.) The choice of the boundary condition $\psi_{z}(0)=0$, as discussed below, is motivated by our requirement that $\hat{p}$ should be symmetric. Because $-\psi_{z}(0)=\zeta(z)$ is the Riemann zeta function, the boundary condition that we have used implies that $z$ must belong to the discrete set of zeros of $\zeta(z)$.

The zeros of the Riemann zeta function may be either trivial or nontrivial. It follows from (4) that for the trivial zeros $z=-2 n \quad(n=1,2,3, \ldots) \quad$ we have $\psi_{z}(x)=$ $-B_{2 n+1}(x+1) /(2 n+1)$, where $B_{n}(x)$ is a Bernoulli polynomial [9]. In this case $\left|\psi_{z}(x)\right|$ grows like $x^{2 n+1}$ as $x \rightarrow \infty$. For the nontrivial zeros $\psi_{z}(x)$ oscillates and $\left|\psi_{z}(x)\right|$ grows sublinearly. In particular, it follows from (4) that for large $x$ we have $\psi_{z}(x) \approx x^{1-z} /(1-z)$. Thus, for the trivial zeros $\hat{\Delta} \psi_{z}(x)$ blows up, but for the nontrivial zeros $\hat{\Delta} \psi_{z}(x)$ goes to zero as $x \rightarrow \infty$. The eigenstates associated with the trivial zeros violate the orthogonality relation discussed below, and the eigenstates associated with the nontrivial zeros do not. These indicate that the eigenstates associated with the trivial zeros do not belong to the domain of $\hat{H}$. Therefore, under the boundary condition $\psi(0)=0$, the $n$th eigenstate of the Hamiltonian (1) is $\psi_{n}(x)=-\zeta\left(z_{n}, x+1\right)$; the eigenvalues $E_{n}=i\left(2 z_{n}-1\right)$ are discrete and $z_{n}=\frac{1}{2}\left(1-i E_{n}\right)$ are the nontrivial zeros of the Riemann zeta function. The Riemann hypothesis is valid if and only if these eigenvalues are real.

The analysis above establishes a complex extended version of the Berry-Keating conjecture [12]. We are not able to prove that the eigenvalues of $\hat{H}$ are real; nevertheless, in what follows we present a heuristic analysis that suggests that the eigenvalues are real. Specifically, we first investigate symmetry properties of $\hat{H}$, which shows that $i \hat{H}$ is $\mathcal{P} \mathcal{T}$ symmetric and $\hat{H}$ is pseudo-Hermitian. This allows us to obtain a quantization of the Berry-Keating Hamiltonian $\hat{h}^{\mathrm{BK}}=\hat{x} \hat{p}+\hat{p} \hat{x}$ that is isospectral to $\hat{H}$. We then make use of the biorthogonality properties of the eigenstates of $\hat{H}$ to introduce an inner product which makes $\hat{H}$ Hermitian.

Relation to pseudo-Hermiticity.-To gain some intuition about the reality of the eigenvalues of the Hamiltonian, we remark first that $i \hat{H}$ is $\mathcal{P} \mathcal{T}$ symmetric $[13,14]$ in the following sense. Under conventional parity-time reflection, if $\hat{p}$ is a momentum and $\hat{x}$ is a coordinate, we have $\mathcal{P} \mathcal{T}:(\hat{x}, \hat{p}) \rightarrow(-\hat{x}, \hat{p})$. However, we consider instead the variables where the roles of position $\hat{x}$ and momentum $\hat{p}$ are interchanged [15]. We then define parity-time reflection as $\mathcal{P} \mathcal{T}:(\hat{x}, \hat{p}) \rightarrow(\hat{x},-\hat{p})$. Therefore, since $\mathcal{P} \mathcal{T}: i \rightarrow-i$, we deduce that $i \hat{H}$ is invariant under this modified $\mathcal{P} \mathcal{T}$ reflection. It follows that the eigenvalues of $i \hat{H}$ are either real (if the $\mathcal{P} \mathcal{T}$ symmetry is unbroken in the sense that the associated eigenstates are also eigenstates of $\mathcal{P} \mathcal{T}$ ), or else they form complex-conjugate pairs (if the $\mathcal{P} \mathcal{T}$ symmetry is broken in the sense that the associated eigenstates are not eigenstates of $\mathcal{P} \mathcal{T}$ ). If the $\mathcal{P} \mathcal{T}$ symmetry is maximally broken for $i \hat{H}$, then the eigenvalues of $\hat{H}$ would be real, and the Riemann hypothesis would hold. In our case, since $\mathcal{P} \mathcal{T} \psi_{n}(x)=\psi_{-n}(x)$, the $\mathcal{P} \mathcal{T}$ symmetry is indeed broken for all complex values of $z_{n}$. (For the trivial zeros the $\mathcal{P} \mathcal{T}$ symmetry is unbroken.)

Let us now assume that the momentum operator $\hat{p}$ is Hermitian (symmetric); that is, the action of $\hat{p}^{\dagger}$ agrees with that of $\hat{p}$ on the domain of $\hat{H}$. Here $\dagger$ denotes the adjoint with respect to the standard inner product on $\mathcal{L}^{2}\left(\mathbb{R}^{+}\right)$. Then the Hermitian adjoint of $\hat{H}$ is

$$
\hat{H}^{\dagger}=\left(\mathbb{1}-e^{i \hat{p}}\right)(\hat{x} \hat{p}+\hat{p} \hat{x}) \frac{\mathbb{1}}{\mathbb{1}-e^{i \hat{p}}} .
$$

Therefore, if we define the operator $\hat{\eta}$ according to

$$
\hat{\eta}=\sin ^{2} \frac{1}{2} \hat{p},
$$

which is non-negative, bounded, and Hermitian under the assumption, we get $\hat{H}^{\dagger}=\hat{\eta} \hat{H} \hat{\eta}^{-1}$; i.e., $\hat{H}$ is pseudo-Hermitian in the sense of [16]. Assuming that $\hat{p}$ is Hermitian, there exists an associated Hermitian Hamiltonian $\hat{h}$ obtained by conjugating $\hat{H}$ with an operator $\hat{\rho}$ satisfying $\hat{\rho}^{\dagger} \hat{\rho}=\hat{\eta}$, that is, $\hat{\rho} \hat{H} \hat{\rho}^{-1}=\hat{h}$. Letting $\hat{\rho}=\sin \frac{1}{2} \hat{p}$, we obtain $\hat{h}=\hat{x} \hat{p}+\hat{p} \hat{x}+\hbar \hat{p}$. We include Planck's constant $\hbar$ explicitly here because it indicates that the linear momentum term is a quantum anomaly; this term vanishes in the classical limit $\hbar \rightarrow 0$ [15]. Alternatively, by letting $\hat{\rho}=\hat{\Delta}$ we obtain the BerryKeating Hamiltonian $\hat{h}^{\mathrm{BK}}=\hat{x} \hat{p}+\hat{p} \hat{x}$, whose eigenstates are $\phi_{z}^{\mathrm{BK}}(x)=x^{-z}$.

The associated Hamiltonian $\hat{h}$ is unique up to unitary transformations, so there are infinitely many formally Hermitian Hamiltonians that are similar to $\hat{H}$ [12]. If both $\hat{\eta}$ and $\hat{\eta}^{-1}$ are positive, bounded, and Hermitian, then the Hamiltonians $\hat{H}$ and $\hat{h}$ are isospectral [17]. Assuming that $\hat{p}$ is Hermitian, these operators are indeed Hermitian and nonnegative, but $\hat{\eta}^{-1}$ is not bounded. Nevertheless, we can show by a direct calculation that $\hat{H}$ and $\hat{h}$ are in fact isospectral. Furthermore, since the map from the eigenstates $\left\{\psi_{n}(x)\right\}$ of $\hat{H}$ to the eigenstates $\left\{\phi_{n}(x)\right\}$ of $\hat{h}$ is governed by $\hat{\rho}$, we can identify the quantization condition for the eigenstates of the associated Hamiltonians explicitly by using the relation $2 i \sin \frac{1}{2} \hat{p} \psi_{z}(x)=\psi_{z}\left(x+\frac{1}{2}\right)-\psi_{z}\left(x-\frac{1}{2}\right)$. For the BerryKeating Hamiltonian, the condition $\psi_{z}(0)=0$ leads to

$$
\lim _{x \rightarrow 0}\left[\phi_{z}^{\mathrm{BK}}(x)-\zeta(z, x-1)\right]=0,
$$

or, equivalently, $\lim _{x \rightarrow 1} \phi_{z}^{\mathrm{BK}}(x)=-\lim _{x \rightarrow 1} \zeta(z, x+1)$. 
Biorthogonal states.-Let us proceed under the assumption that $\hat{p}$ is Hermitian. Because $\hat{H}$ is not Hermitian, its eigenstates $\left\{\psi_{n}(x)\right\}$ are not orthogonal. Nevertheless, by considering the eigenstates $\left\{\tilde{\psi}_{n}(x)\right\}$ of $\hat{H}^{\dagger}$ we obtain a biorthogonal set of eigenstates [17], provided that $\hat{H}^{\dagger}$ is the Hermitian adjoint of $\hat{H}$. Bearing in mind that $\hat{\Delta}^{\dagger}$ is the forward difference operator, a calculation shows that $\tilde{\psi}_{n}(x)=x^{-z_{n}}-(x+1)^{-z_{n}}$ and that $\hat{H}^{\dagger} \tilde{\psi}_{n}(x)=i\left(2 z_{n}-1\right) \tilde{\psi}_{n}(x)$. Using $\left\{\tilde{\psi}_{n}(x)\right\}$, we introduce an inner product on the space of functions spanned by $\left\{\psi_{n}(x)\right\}$ as follows. For any $\psi(x)=\sum_{n} c_{n} \psi_{n}(x)$ we define its associated state by $\tilde{\psi}(x)=\sum_{n} c_{n} \tilde{\psi}_{n}(x)$. The inner product of a pair of such functions $\psi(x)$ and $\varphi(x)$ is then defined by $\langle\varphi, \psi\rangle=\langle\tilde{\varphi} \mid \psi\rangle:=\int_{0}^{\infty} \overline{\tilde{\varphi}(x)} \psi(x) d x$. Alternatively stated, since $\tilde{\varphi}(x)=\hat{\eta} \varphi(x)$, we have $\langle\varphi, \psi\rangle=\langle\varphi|\hat{\eta}| \psi\rangle$; that is, the positive Hermitian operator $\hat{\eta}$ plays the role of the metric (or, equivalently, the $\mathcal{C P}$ operator [18]).

For $\hat{H}$ in (1) the inner-product space constructed above is not a Hilbert space because, as we will see, the elements of the vector space have infinite norm. However, the elements of $\left\{\psi_{n}(x)\right\}$ and those of $\left\{\tilde{\psi}_{n}(x)\right\}$ are biorthogonal provided that $\left\{z_{n}\right\}$ belongs to the nontrivial zeros of the Riemann zeta function. To see this, let us consider the inner product $\left\langle\tilde{\psi}_{m} \mid \psi_{n}\right\rangle$. Observing that

$$
\tilde{\psi}_{m}(x)=\hat{\Delta}^{\dagger} \hat{\Delta} \psi_{n}(x)=\hat{\Delta}^{\dagger} \hat{\Delta} \hat{\Delta}^{-1} x^{-z_{m}}=\hat{\Delta}^{\dagger} x^{-z_{m}},
$$

and recalling that $\psi_{n}(x)=\hat{\Delta}^{-1} x^{-z}$, we find that

$$
\begin{aligned}
\left\langle\tilde{\psi}_{m} \mid \psi_{n}\right\rangle & =\int_{0}^{\infty} d x x^{-\bar{z}_{m}} \hat{\Delta} \hat{\Delta}^{-1} x^{-z_{n}} \\
& =\int_{0}^{\infty} d x x^{-1+i\left(E_{n}-\bar{E}_{m}\right) / 2} .
\end{aligned}
$$

Thus, if $\bar{E}_{m}=E_{m}$ (that is, if the Riemann hypothesis is correct), then (6) is a Dirac delta function $4 \pi \delta\left(E_{n}-E_{m}\right)$. It follows that for $m \neq n$ we have

$$
\left\langle\tilde{\psi}_{m} \mid \psi_{n}\right\rangle=0
$$

in the distributional sense, as required by the biorthogonality condition. In contrast, for the trivial zeros, the integral (6) diverges too rapidly to be interpreted as a tempered distribution.

In terms of the inner product introduced above, and assuming that $\hat{p}$ is Hermitian (symmetric), we find, using $\hat{\Delta}^{\dagger} \hat{\Delta}=\hat{\eta}$, that

$$
\begin{aligned}
\langle\hat{H} \varphi, \psi\rangle & =\int_{0}^{\infty} d x \bar{\varphi}(x) \hat{\Delta}^{\dagger}(\hat{x} \hat{p}+\hat{p} \hat{x})\left(\hat{\Delta}^{\dagger}\right)^{-1} \hat{\Delta}^{\dagger} \hat{\Delta} \psi(x) \\
& =\int_{0}^{\infty} d x \bar{\varphi}(x) \hat{\Delta}^{\dagger} \hat{\Delta} \hat{\Delta}^{-1}(\hat{x} \hat{p}+\hat{p} \hat{x}) \hat{\Delta} \psi(x) \\
& =\langle\varphi, \hat{H} \psi\rangle .
\end{aligned}
$$

This shows that, from the assumption that $\hat{p}$ is Hermitian, we may conclude that $\hat{H}$ is Hermitian (symmetric) with respect to the new inner product.

As a further consequence of (6) and (7), if the Riemann hypothesis is true, then the eigenvalues of $\hat{H}$ are nondegenerate. Conversely, if the Riemann hypothesis is false, then the eigenstates of $\hat{H}$ that correspond to nontrivial zeros for which $\operatorname{Re}(z) \neq \frac{1}{2}$ coalesce to give rise to Jordan block structures in the Hamiltonian. This follows from the fact that at such complex degeneracies (often referred to as exceptional points), the eigenstates satisfy the so-called self-orthogonality condition $\left\langle\tilde{\psi}_{n} \mid \psi_{n}\right\rangle=0$. These findings may have an implication on whether the zeros of $\zeta(z)$ are simple: It is known that if the Riemann hypothesis holds true, then at least 19/27 of the nontrivial zeros are simple [19]. However, if there exists a one-to-one correspondence between the boundary condition on the eigenstates of $\hat{H}$ and the secular equation for the eigenvalues of $\hat{H}$, then it follows that the validity of the Riemann hypothesis implies that all roots are simple, and conversely any nontrivial zero of $\zeta(z)$ for which $\operatorname{Re}(z) \neq \frac{1}{2}$ cannot be simple.

Boundary condition revisited.-For finite-dimensional nondegenerate matrices, the biorthogonality relation (7) implies that $\hat{H}^{\dagger}$ defined in (5) is the Hermitian adjoint of $\hat{H}$. However, in infinite-dimensional vector spaces the completeness of the states $\left\{\psi_{n}(x)\right\}$ is required to arrive at this conclusion. Nevertheless, the relation (7) suggests that our Hermiticity assumption of $\hat{p}$ is valid, making $\hat{h}$ manifestly Hermitian.

Encouraged by this observation, we ask whether the momentum operator $\hat{p}$ is Hermitian (symmetric) on the inner-product space defined above. Because $[\hat{p}, \hat{\eta}]=0$, the Hermiticity of $\hat{p}$ on $\langle\cdot, \cdot\rangle$ follows if the boundary terms vanish under an integration by parts when the elements of $\left\{\psi_{n}(x)\right\}$ and those of $\left\{\tilde{\psi}_{n}(x)\right\}$ are paired. Note that $\tilde{\psi}_{n}(x)$ diverges at $x=0$, so $\psi_{n}(x)$ must vanish sufficiently fast at $x=0$ to ensure the vanishing of the boundary terms. [The divergence of $\left\{\psi_{n}(x)\right\}$ at $x=\infty$ is compensated by the vanishing of $\left\{\tilde{\psi}_{n}(x)\right\}$ as $x \rightarrow \infty$.] One can verify that imposing $\psi_{n}(0)=0$ is sufficient to guarantee the vanishing of the boundary term at the origin. Thus, the Hermiticity of $\hat{p}$ on $\langle\cdot, \cdot\rangle$ follows from the boundary condition $\psi_{n}(0)=0$.

Relation to quantum mechanics.-Since the operator $\hat{H}$ is a function of the canonical variables $(\hat{x}, \hat{p})$, we have referred to it as a Hamiltonian. However, the connection of this Hamiltonian to physical systems is at best tenuous because the eigenstates of $\hat{H}$ in our inner-product space are not normalizable. This is not a concern for our analysis, but in quantum mechanics normalizability is required for a probabilistic interpretation.

A possible way of making a connection to quantum theory is to introduce a regularization scheme, for example, by letting $x \in\left[\Lambda^{-1}, \Lambda\right]$, renormalizing the states according to $\psi_{n}(x) \rightarrow(\ln \Lambda)^{-1 / 2} \psi_{n}(x)$, and then taking the limit 
$\Lambda \rightarrow \infty$. Interestingly, the expectation value of the position operator $\hat{\rho}^{-1} \hat{x} \hat{\rho}$ in the state $\psi_{n}(x)$ for any $n$ in the renormalized theory is $\Lambda / \ln \Lambda$, which for large $\Lambda$ gives the leading term in the counting of prime numbers smaller than $\Lambda$.

Discussion.-We have presented a formal argument showing that the eigenvalues of the Hamiltonian $\hat{H}$ in (1), whose classical limit is $2 x p$, correspond to the nontrivial zeros of the Riemann zeta function. Identifying the domain of $\hat{H}$ remains a difficult and open problem. We hope that further analysis of the properties of $\hat{H}$, such as identifying its domain and establishing its self-adjointness, will prove the reality of the eigenvalues, and thus the veracity of the Riemann hypothesis. The possibility of extending the Hilbert-Pólya program to non-Hermitian $\mathcal{P} \mathcal{T}$-symmetric operators has been noted [20]. We hope that our findings will significantly boost research in this direction. The fact that $i \hat{H}$ is $\mathcal{P} \mathcal{T}$ symmetric, with a broken $\mathcal{P} \mathcal{T}$ symmetry, offers a fresh and optimistic outlook.

D. C. B. thanks D. Blasius and C. Hughes for comments and the Russian Science Foundation for support (Project No. 16-11-10218). M. P. M. thanks D. Schleicher for discussions. M. P. M. is supported in part by the Canada Research Chairs program. Research at Perimeter Institute is supported by the Government of Canada through Innovation, Science and Economic Development Canada and by the Province of Ontario through the Ministry of Research, Innovation and Science.

[1] B. Riemann, Ueber die Anzahl der Primzahlen unter einer gegebenen Grösse, Monatsberichte der Berliner Akademie (Monatsberichte der Königlichen Preußischen Akademie der Wissenschaften zu Berlin, Berlin, 1859).

[2] H. L. Montgomery, in Analytic Number Theory. Proceedings of the Symposium on Pure Mathematics XXIV (American Mathematical Society, Providence, 1973), pp. 181-193.

[3] A. M. Odlyzko, On the distribution of spacings between zeros of the zeta function, Math. Comput. 48, 273 (1987).

[4] M. V. Berry, in Quantum Chaos and Statistical Nuclear Physics, edited by T. H. Seligman and H. Nishioka, Lect. Notes Phys. Vol. 263 (Springer-Verlag, New York, 1986).

[5] M. V. Berry and J. P. Keating, in Supersymmetry and Trace Formulae: Chaos and Disorder, edited by I. V. Lerner et al. (Kluwer Academic/Plenum, New York, 1999) [http://link .springer.com/chapter/10.1007\%2F978-1-4615-4875-1_19].
[6] A. Connes, Trace formula in noncommutative geometry and the zeros of the Riemann zeta function, Sel. Math. New Ser. 5, 29 (1999).

[7] G. Sierra, The Riemann zeros as spectrum and the Riemann hypothesis, arXiv:1601.01797.

[8] Ë. Delabaere, Ramanujan's summation, Algorithms Sem. 2001-2002, 83 (2003) [http://algo.inria.fr/seminars/ sem01-02/delabaere2.pdf].

[9] F. W. J. Olver, D. M. Lozier, R. F. Boisvert, and C. W. Clark, NIST Handbook of Mathematical Functions (Cambridge University Press, Cambridge, England, 2010).

[10] C. M. Bender and S. A. Orszag, Advanced Mathematical Methods for Scientists and Engineers (McGraw-Hill, New York, 1978).

[11] M. Müller and D. Schleicher, How to add a non-integer number of terms, and how to produce unusual infinite summations, J. Comput. Appl. Math. 178, 347 (2005); Fractional sums and Euler-like identities, Ramanujan J. 21, 123 (2010); How to add a noninteger number of terms: From axioms to new identities, Am. Math. Mon. 118, 136 (2011).

[12] One can extend $\hat{H}$ to a one-parameter family of Hamiltonians $\hat{H}_{\varepsilon}$ by the replacement $\hat{\Delta} \rightarrow \hat{\Delta}_{\varepsilon}=\varepsilon^{-1}\left(\mathbb{1}-e^{-i \varepsilon \hat{p}}\right)$. A calculation shows that the eigenstates $\psi_{z}^{\varepsilon}(x)$ of $\hat{H}_{\varepsilon}$ take the form $\psi_{z}^{\varepsilon}(x) \propto-\zeta(z, 1+x / \varepsilon)$ with eigenvalue $i(2 z-1)$. In the limit $\varepsilon \rightarrow 0$ we obtain the Hamiltonian $\hat{p}^{-1}(\hat{x} \hat{p}+\hat{p} \hat{x}) \hat{p}$ with eigenstate $x^{1-z}$.

[13] C. M. Bender, Making sense of non-Hermitian Hamiltonians, Rep. Prog. Phys. 70, 947 (2007).

[14] D. C. Brody, Consistency of PT-symmetric quantum mechanics, J. Phys. A 49, 10LT03 (2016).

[15] C. M. Bender, D. C. Brody, J.-H. Chen, H. F. Jones, K. A. Milton, and M.C. Ogilvie, Equivalence of a complex PT-Symmetric quartic Hamiltonian and a Hermitian quartic Hamiltonian with an anomaly, Phys. Rev. D 74, 025016 (2006).

[16] G. W. Mackey, Commutative Banach Algebras (Instituto de Matematica pura e Aplicada do Conselho Nacional de Pesquisa, Rio De Janeiro, 1959).

[17] D. C. Brody, Biorthogonal quantum mechanics, J. Phys. A 47, 035305 (2014).

[18] C. M. Bender, D. C. Brody, and H. F. Jones, Complex Extension of Quantum Mechanics, Phys. Rev. Lett. 89, 270401 (2002).

[19] H. M. Bui and D. R. Heath-Brown, On simple zeros of the Riemann zeta-function, Bull. London Math. Soc. 45, 953 (2013).

[20] Z. Ahmed and S. R. Jain, A pseudo-unitary ensemble of random matrices, PT-symmetry and the Riemann hypothesis, Mod. Phys. Lett. A 21, 331 (2006). 